\title{
Fixed point theorems for $w$-cone distance contraction mappings in tvs-cone metric spaces
}

\author{
Ljubomir Ćirić $^{*}$, Hossein Lakzian ${ }^{2}$ and Vladimir Rakočević ${ }^{3}$
}

\footnotetext{
* Correspondence: Iciric@rcub.bg. ac.rs

${ }^{1}$ Faculty of Mechanical Engineering, Kraljice Marije 16, Belgrade, Serbia Full list of author information is available at the end of the article
}

\begin{abstract}
In this article, we introduce the concept of a $w$-cone distance on topological vector space (tvs)-cone metric spaces and prove various fixed point theorems for $\mathrm{w}$-cone distance contraction mappings in tvs-cone metric spaces. The techniques of the proof of our theorems are more complex then in the corresponding previously published articles, since a new technique was necessary for the considered class of mappings. Presented fixed point theorems generalize results of Suzuki and Takahashi, Abbas and Rhoades, Pathak and Shahzad, Raja and Veazpour, Hicks and Rhoades and several other results existing in the literature.

Mathematics subject classification (2010): 47H10; 54H25.
\end{abstract}

Keywords: fixed point, w-distance, tvs cone metric

\section{Introduction and preliminaries}

There exist many generalizations of the concept of metric spaces in the literature. Fixed point theory in abstract metric or $K$-metric spaces was developed in the middle of 70th years of twentieth century. Huang and Zhang [1] re-introduced and studied the concept of cone metric spaces over a Banach space, and proved several fixed point theorems. Then, there have been a lot of articles in which known fixed point theorems in metric are extended to cone metric spaces. Recently, Du [2] used the scalarization function and investigated the equivalence of vectorial versions of fixed point theorems in $K$-metric spaces and scalar versions of fixed point theorems in metric spaces. He showed that many of the fixed point theorems for mappings satisfying contractive conditions of a linear type in $K$-metric spaces can be considered as corollaries of corresponding theorems in metric spaces. Nevertheless, the fixed point theory in $K$-metric spaces proceeds to be actual, since the method of scalarization function cannot be applied for a wide class of weakly contractive mapping, satisfying nonlinear contractive conditions.

Kada et al. [3] introduced and studied the concept of $w$-distance on a metric space. They give examples of $w$-distance and improved Caristi's fixed point theorem, Ekeland's $\varepsilon$-variational's principle and the nonconvex minimization theorem according to Takahashi (see many useful examples and results on $w$-distance in [4-8] and in references there in).

Definition 1. [3]. Let $X$ be a metric space with metric $d$. Then a function $p: X \times X$ $\rightarrow[0, \infty)$ is called $a w$-distance on $X$ if the following are satisfied:

(C) 2012 Ćirićć et al; licensee Springer. This is an Open Access article distributed under the terms of the Creative Commons Attribution License (http://creativecommons.org/licenses/by/2.0), which permits unrestricted use, distribution, and reproduction in any medium, provided the original work is properly cited. 
(1) $p(x, z) \leq p(x, y)+p(y, z)$, for any $x, y, z \in X$,

(2) for any $x \in X, p(x, \cdot): X \rightarrow[0, \infty)$ is lower semicontinuous,

(3) for any $\varepsilon>0$, there exist $\delta>0$ such that $p(z, x) \leq \delta$ and $p(z, y) \leq \delta$ imply $d(x, y) \leq \varepsilon$.

In the following we suppose that $E$ is a real Hausdorff topological vector space ( $t v s$ for short) with the zero vector $\theta$. A proper nonempty and closed subset $P$ of $E$ is called a (convex) cone if $P+P \subset P, \lambda P \subset P$ for $\lambda \geq 0$ and $P \cap(-P)=\theta$. We shall always assume that the cone $P$ has a nonempty interior int $P$ (such cones are called solid).

Each cone $P$ induces a partial order $\preccurlyeq$ on $E$ by $x \leqslant y \Leftrightarrow y-x \in P$. $x \prec y$ will stand for $x \leqslant y$ and $x \neq y$, while $x \ll y$ will stand for $y-x \in$ int $P$. The pair $(E, P)$ is an ordered tvs.

Let us recall that the algebraic operations in tvs-cone are continuous functions. For the convenience of the reader we give the next result.

Lemma 2. Let $E$ be a tvs over $\mathscr{F}=\mathbb{R}, \mathbb{C}$.

(1) Suppose that $x_{n}, y_{n}, x, y \in E$ and $x_{n} \rightarrow x$ and $y_{n} \rightarrow y$. Then $x_{n}+y_{n} \rightarrow x+y$.

(2) Suppose that $x_{n}, x \in E, \lambda_{n}, \lambda \in \mathscr{F}, x_{n} \rightarrow x$ and $\lambda_{n} \rightarrow \lambda$. Then $\lambda_{n} x_{n} \rightarrow \lambda_{x}$.

Proof. (1) Suppose that $W \subset E$ is an open set and $x+y \in W$. Let us define $f: E \times E$ $\mapsto E$ by $f(u, v)=u+v, u, v \in E$. Because $f$ is continuous at $(x, y)$ there is an open set $G \subset E \times E$ such that $(x, y) \in G$ and $f(G) \subset W$. Now there are open sets $U_{i}, V_{i} \subset E, i \in$ $I$, such that $G=\cup_{i L I} U_{i} \times V_{i}$. Hence, there exists $i_{0} \in I$ such that $(x, y) \in U_{i_{0}} \times V_{i_{0}}$. Because $x \in U_{i_{0}}$ and $x_{n} \rightarrow x$, there exists $n_{1}$ such that $x_{n} \in U_{i_{0}}$ for all $n \geq n_{1}$. Also, because $y \in V_{i_{0}}$ and $y_{n} \rightarrow y$, there exists $n_{2}$ such that $y_{n} \in V_{i_{0}}$ for all $n \geq n_{2}$. Hence, $x_{n}+y_{n}=f\left(x_{n}, y_{n}\right) \in f\left(U_{i_{0}} \times V_{i_{0}}\right) \subset W$ for all $n \geq \max \left\{n_{1}, n_{2}\right\}$. Thus, $x_{n}+y_{n} \rightarrow x+y$.

(2) Suppose that $W \subset E$ is an open set and $\lambda x \in W$. Let us define $g: \mathscr{F} \times \mathscr{E} \mapsto \mathscr{E}$ by $g$ $(\mu, v)=\mu \nu, \mu \in \mathscr{F}, v \in \mathscr{E}$. Because $g$ is continuous at $(\lambda, x)$ there is an open set $G \subset \mathscr{F}$ $\times \mathscr{E}$ such that $(\lambda, x) \in G$ and $g(G) \subset W$. Now there are open sets $U_{i} \subset \mathscr{F}, V_{i} \subset \mathscr{E}, i \in$ $I$, such that $G=\cup{ }_{i L I} U_{i} \times V_{i}$. Hence, there exists $i_{0} \in I$ such that $(\lambda, x) \in U_{i_{0}} \times V_{i_{0}}$. Because $\lambda \in U_{i_{0}}$ and $\lambda_{n} \rightarrow \lambda$, there exists $n_{1}$ such that $\lambda_{n} \in U_{i_{0}}$ for all $n \geq n_{1}$. Also, because $x \in V_{i_{0}}$ and $x_{n} \rightarrow x$, there exists $n_{2}$ such that $x_{n} \in V_{i_{0}}$ for all $n \geq n_{2}$. Hence, $\lambda_{n} x_{n}=g\left(\lambda_{n}, x_{n}\right) \in g\left(U_{i_{0}} \times V_{i_{0}}\right) \subset W$ for all $n \geq \max \left\{n_{1}, n_{2}\right\}$. Thus, $\lambda_{n} x_{n} \rightarrow \lambda x$.

Following $[1,2,9,10]$ we give the following

Definition 3. Let $X$ be a nonempty set and (E.P) an ordered tvs. A function $d: X \times X$ $\rightarrow E$ is called a tvs-cone metric and $(X, d)$ is called a tvs-cone metric space if the following conditions hold:

(C1) $\theta \leqslant d(x, y)$ for all $x, y \in X$ and $d(x, y)=\theta$ if and only if $x=y$;

(C2) $d(x, y)=d(y, x)$ for all $x, y \in X$;

(C3) $d(x, z) \leqslant d(x, y)+d(y, z)$ for all $x, y, z \in X$.

Let $x \in X$ and $\left\{x_{n}\right\}$ be a sequence in $X$. Then, it is said that

(i) $\left\{x_{n}\right\}$ (tvs-cone) converges to $x$ if for every $c \in E$ with $\theta \ll c$ there exists a natural number $n_{0}$ such that $d\left(x_{n}, x\right) \ll c$ for all $n>n_{0}$; we denote it by $\lim _{n \rightarrow \infty} \mathrm{x}_{\mathrm{n}}=x$ or $x_{n}$ $\rightarrow x$ as $n \rightarrow \infty$;

(ii) $\left\{x_{n}\right\}$ is a (tvs-cone) Cauchy sequence if for every $c \in E$ with $0 \ll c$ there exists a natural number $n_{0}$ such that $d\left(x_{m}, x_{n}\right) \ll c$ for all $m, n>n_{0}$;

(iii) $(X, d)$ is (tvs-cone) complete if every tvs-Cauchy sequence is (tvs) convergent in $X$.

Let $(X, d)$ be a tvs-cone metric space. Then the following properties are often used (see e.g., $[1,2,9-13])$.

$\left(\mathbf{p}_{1}\right)$ If $u \leq v$ and $v \ll w$ then $u \ll w$. 
$\left(\mathbf{p}_{2}\right)$ If $a \leq b+c$ for each $c \in$ int $P$ then $a \leq b$.

$\left(\mathbf{p}_{3}\right)$ If $a \leq \lambda a$, where $a \in P$ and $0<\lambda<1$, then $a=\theta$.

$\left(\mathbf{p}_{4}\right)$ If $\varepsilon \in$ int $P, \theta \leq a_{n}$ and $a_{n} \rightarrow \theta$, then there exists $n_{0}$ such that for all $n>n_{0}$ we have $a_{n} \ll \varepsilon$.

\section{$\mathbf{2} \mathbf{w}$-Cone distance in tvs-cone metric spaces}

Let $(X, d)$ be a tvs-cone metric space. Then

$\left(\mathbf{c}_{1}\right) T: X \rightarrow X$ is continuous at $x \in X$ if $x_{n}$ is a sequence in $X$ and $\lim x_{n}=x$ implies $T(x)=\lim T\left(x_{n}\right)$.

$\left(\mathbf{c}_{2}\right) G: X \rightarrow P$ is lower semicontinuous at $x \in X$ if for any $\varepsilon$ in $E$ with $\theta \ll \varepsilon$, there is $n_{0}$ in $\mathbb{N}$ such that

$$
G(x) \leq G\left(x_{n}\right)+\varepsilon, \quad \text { for all } n \geq n_{0},
$$

whenever $\left\{x_{n}\right\}$ is a sequence in $X$ and $x_{n} \rightarrow x$.

$\left(\mathbf{c}_{3}\right)$ For $x \in X, T: X \rightarrow X, O(x ; \infty)=\left\{x, T x, T^{2} x, \ldots\right\}$ is called the orbit of $x . G: X \rightarrow$ $P$ is

$T$-orbitally lower semicontinuous at $x$ if for any $\varepsilon$ in $E$ with $\theta \ll \varepsilon$, there is $n_{0}$ in $\mathbb{N}$ such that (1) with $x=u$ holds whenever $x_{n} \in O(x ; \infty)$ and $x_{n} \rightarrow u$.

Observe that if in definitions $\left(\mathbf{c}_{1}\right),\left(\mathbf{c}_{2}\right)$ and $\left(\mathbf{c}_{3}\right)$ we have $E=\mathbb{R}, P=[0, \infty),\|x\|=|x|$, $x \in E$, then we get the well-known definitions of continuity, lower and $T$-orbitally lower semicontinuity.

Definition 4. Let $(X, d)$ be a tvs-cone metric space. Then, a function $p: X \times X \rightarrow P$ is called a w-cone distance on $X$ if the following are satisfied:

$\left(\boldsymbol{w}_{1}\right) p(x, z) \leq p(x, y)+p(y, z)$, for any $x, y, z \in X$,

$\left(\boldsymbol{w}_{2}\right)$ for any $x \in X, p(x, \cdot): X \rightarrow P$ is lower semicontinuous,

$\left(\boldsymbol{w}_{3}\right)$ for any $\varepsilon$ in $E$ with $\theta \ll \varepsilon$, there is $\delta$ in $E$ with $\theta \ll \delta$, such that $p(z, x) \ll \delta$ and $p(z, y) \ll \delta$ imply $d(x, y) \ll \varepsilon$.

Example 5. Let $(X, d)$ be a cone metric space. Then, a cone metric $d$ is a w-cone distance $p$ on $X$.

Proof. Clearly, if $p=d$, a $w$-cone distance $p$ satisfies $\left(\mathbf{w}_{1}\right)$ and $\left(\mathbf{w}_{3}\right)$. So we have only to prove $\left(\mathbf{w}_{2}\right)$. Suppose that $x, y \in X, y_{n} \in X, y_{n} \rightarrow y$ and $\varepsilon$ in $E$ with $\theta \ll \varepsilon$ be arbitrary. Since $y_{n} \rightarrow y$, then there is $n_{0}$ in $\mathbb{N}$ such that $d\left(y_{n}, y\right) \ll \varepsilon$ for all $n \geq n_{0}$. Define $G(y)=d(x, y)$. Then we have

$$
G(y)=d(x, y) \leq d\left(x, y_{n}\right)+d\left(y_{n}, y\right) \leq d\left(x, y_{n}\right)+\varepsilon=G\left(y_{n}\right)+\varepsilon
$$

for all $n \geq n_{0}$. Therefore $p(x, \cdot)=d(x, \cdot)$ is lower semicontinuous.

Remark 6. Wang and Guo [14]defined the concept of c-distance on a cone metric space in the sense of Huang and Zhang [1], which is also a generalization of $w$-distance of Kada et al. [3]. They proved a common fixed point theorem (Theorem 2.2) by using $c$-distance in a cone metric space $(X, d)$, where a cone $P$ is normal with normal constant $K$. Now we shall present an example (Example 7 below), which shows that there are cone metric spaces where underlying cone is not normed, and so theorems of Wang and Guo [14]cannot be applied. On the other case, our presented fixed point theorems for mappings under contractive conditions expressed in the terms of $w$-distance can be applied, although the underlying cone is not normed. 
Example 7. Let $E=C[0,1]$ be the Banach space of real-valued continuous functions with the usual norm

$$
|| f(t)-g(t) \|=\max _{0 \leq t \leq 1}|f(t)-g(t)|
$$

and let a cone $P$ be defined by $P=\{f \in E: f(t) \geq 0$ for $t \in[0,1]\}$. This cone is normal in the Banach-space topology on E. Let $\tau^{*}$ be the strongest locally convex topology on the linear vector space $E$. Then, intP $\neq \varnothing$, but the cone $P$ is not normal in the topology $\tau^{*}$. Indeed, if we suppose, to the contrary, that $P$ is normal cone, then the topology $\tau^{*}$ is normed (see, e.g., [15]). But if the cone of an ordered tvs is solid and normal, then such tvs must be an ordered normed space, which is impossible because an infinite dimensional space with the strongest locally convex topology cannot be metrizable (see, e.g., [13]). Let now $X=[0,+\infty)$ and $d: X \times X \rightarrow\left(E, \tau^{*}\right)$ be defined by $d(x, y)(t)=|x-y| \cdot e^{t}$. Then $(X, d)$ is a tvs-cone metric space over the non normedzable linear tvs $\left(E, \tau^{*}\right)$.

The following lemma is crucial and is an extension of Lemma 1 in [3] for a $w$-metric distance to a $w$-cone distance.

Lemma 8. Let $(X, d)$ be a tvs-cone metric space and let $p$ be a w-cone distance on $X$. Let $\left\{x_{n}\right\}$ and $\left\{y_{n}\right\}$ be sequences in $X$, let $\left\{\alpha_{n}\right\}$ with $\theta \leq \alpha_{n}$, and $\left\{\beta_{n}\right\}$ with $\theta \leq \beta_{n}$, be sequences in $E$ converging to $\theta$, and let $x, y, z \in X$. Then the following hold:

(i) If $p\left(x_{n}, y\right) \leq \alpha_{n}$ and $p\left(x_{n}, z\right) \leq \beta_{n}$ for any $n \in \mathbb{N}$, then $y=z$. In particular, if $p(x, y)$ $=\theta$

and $p(x, z)=\theta$, then $y=z$.

(ii) If $p\left(x_{n}, y_{n}\right) \leq \alpha_{n}$ and $p\left(x_{n}, z\right) \leq \beta_{n}$ for any $n \in \mathbb{N}$, then $\left\{y_{n}\right\}$ converges to $z$.

(iii) If $p\left(x_{n}, x_{m}\right) \leq \alpha_{n}$ for any $n, m \in \mathbb{N}$ with $m>n$, then $\left\{x_{n}\right\}$ is a Cauchy sequence.

(iv) If $p\left(y, x_{n}\right) \leq \alpha_{n}$ for any $n \in \mathbb{N}$, then $\left\{x_{n}\right\}$ is a Cauchy sequence.

Proof. We shall prove (ii). Let $\varepsilon$ in $E$ with $\theta \ll \varepsilon$ be arbitrary. From $\left(\mathbf{w}_{3}\right)$ of Definition 4 there is $\delta$ in $E$ with $\theta \ll \delta$, such that $p\left(x_{n}, y_{n}\right) \ll \delta$ and $p\left(y_{n}, z\right) \ll \delta$ imply $d\left(y_{n}\right.$, $z) \ll \varepsilon$. Since $\alpha_{n} \rightarrow \theta$ and $\beta_{n} \rightarrow \theta$, there is $n_{0} \in N$ such that $\alpha_{n} \leq \delta$ and $\beta_{n} \leq \delta$ for all $n \geq n_{0}$. Then for all $n \geq n_{0}$ we have

$$
p\left(x_{n}, y_{n}\right) \leq \alpha_{n} \ll \delta \text { and } p\left(x_{n}, z\right) \leq \beta_{n} \ll \delta .
$$

Thus from $\left(\mathbf{w}_{3}\right), d\left(y_{n}, z\right) \ll \varepsilon$. Hence $y_{n} \rightarrow z$ as $n \rightarrow \infty$. Similarly, following lines of the proof of Lemma 1 in [3], one can prove (i), (iii) and (iv).

\section{Fixed point theorems for $w$-cone distance contraction mappings in $K$ - metric spaces}

We note that the method of $\mathrm{Du}$ [2] for cone contraction mappings cannot be applied for a $w$-cone distance contraction mappings.

In the following theorem, which extends and improves Theorem 2 of [3] and Theorem 1 of [5], we give an estimate for a $w$-cone distance $p\left(x_{n}, z\right)$ of an approximate value $x_{n}$ and a fixed point $z$.

Theorem 9. Let $(X, d)$ be a complete tvs-cone metric space with $w$-cone distance $p$ on $X$. Suppose that for some $0 \leq k<1$ a mapping $T: X \rightarrow X$ satisfies the following condition:

$$
p\left(T x, T^{2} x\right) \leq k p(x, T x), \quad \text { for all } x \in X .
$$


(i) If $y \neq T y$, there exists $c \in \operatorname{int}(P), c \neq \theta$, such that

$$
c \ll p(x, y)+p(x, T x), \text { for all } x \in X ;
$$

(ii) $T$ is continuous.

Then, there exists $z \in X$, such that $z=T z$ and

$$
p\left(T^{n} x, z\right) \leq \frac{k^{n}}{1-k} \cdot p(x, T x) \text { for } n \geq 1 .
$$

Moreover, if $v=T v$ for some $v \in X$, then $p(v, v)=\theta$.

Proof. Let $x \in X$ and define a sequence $\left\{x_{n}\right\}$ by $x_{0}=x ; x_{n}=T^{n} x$ for any $n \in N$. Then from (2) we have, for any $n \in N$,

$$
p\left(x_{n}, x_{n+1}\right)=p\left(T x_{n-1}, T x_{n}\right) \leq k p\left(x_{n-1}, x_{n}\right) \leq \cdots \leq k^{n} p(x, T x) .
$$

Thus, if $m>n$, then from $\left(\mathbf{w}_{1}\right)$ of Definition 4 and (4),

$$
\begin{gathered}
p\left(x_{n}, x_{m}\right) \leq p\left(x_{n}, x_{n+1}\right)+\cdots+p\left(x_{m-1}, x_{m}\right) \\
\leq k^{n} p(x, T x)+\cdots+k^{m-1} p(x, T x) \\
\leq \frac{k^{n}}{1-k} \cdot p(x, T x) .
\end{gathered}
$$

Hence, by (iii) of Lemma 8 with $\alpha_{n}=\left[k^{n} /(1-k)\right] \cdot p(x, T x),\left\{x_{n}\right\}$ is a Cauchy sequence in $X$. Since $X$ is complete, $\left\{x_{n}\right\}$ converges to some point $z \in X$.

Now we shall prove the estimate (3). Define a function $G: X \rightarrow P$ by $G(x)=p\left(x_{n}, x\right)$, where $n$ is any fixed positive integer. Since $x_{n} \rightarrow z$ as $n \rightarrow \infty$, from $\left(\mathbf{w}_{2}\right)$ of Definition 4 and (2) we have that for any $\varepsilon$ in $E$ with $\theta \ll \varepsilon$, there is $m_{0}$ in $\mathbb{N}$ such that

$$
p\left(x_{n}, z\right) \leq p\left(x_{n}, x_{m}\right)+\varepsilon \text { for } m>m_{0} .
$$

Thus by (5) we get

$$
p\left(x_{n}, z\right) \leq \frac{k^{n}}{1-k} \cdot p(x, T x)+\varepsilon .
$$

Hence, as $x_{n}=T^{n} x$,

$$
p\left(T^{n} x, z\right) \leq \frac{k^{n}}{1-k} \cdot p(x, T x)+\varepsilon \quad \text { for any } \varepsilon \text { in } E \text { with } \theta \ll \varepsilon .
$$

Thus, taking $\varepsilon=\varepsilon / i$ we have

$$
p\left(T^{n} x, z\right) \leq \frac{k^{n}}{1-k} \cdot p(x, T x)+\frac{\varepsilon}{i} \text { for each } i \geq 1 .
$$

From (7) and by definition of the partial order $\leq$ on $E$, we obtain

$$
\frac{\varepsilon}{i}-p\left(T^{n} x, z\right)+\frac{k^{n}}{1-k} \cdot p(u, T u) \in P .
$$

By Lemma 2, it is easy to show that

$$
\lim _{i \rightarrow \infty}\left[\frac{\varepsilon}{i}-p\left(T^{n} x, z\right)+\frac{k^{n}}{1-k} \cdot p(u, T u)\right]=-p\left(T^{n} x, z\right)+\frac{k^{n}}{1-k} \cdot p(u, T u) .
$$


Therefore, as $P$ is closed,

$$
-p\left(T^{n} x, z\right)+\frac{k^{n}}{1-k} \cdot p(x, T x) \in P .
$$

From the definition of partial order $\leq$, (8) is equivalent to (3). Thus we proved (3).

Suppose that the case (i) is satisfied. We have to prove that $T z=z$. Suppose, to the contrary, that $z \neq T z$. Then from (i) there exists $c \in \operatorname{int}(P)$ such that

$$
c \ll p(x, z)+p(x, T x), \text { forall } x \in X .
$$

From (6) and (3) we conclude that there exists $n_{0} \in \mathbb{N}$ such that

$$
p\left(x_{n}, z\right) \ll \frac{c}{4} \text { and } p\left(x_{n}, x_{n+1}\right) \ll \frac{c}{4} \text { for all } n>n_{0} .
$$

Since $x_{n} \rightarrow z$ as $n \rightarrow \infty$, by (ii) of Definition 4 with $x=x_{n_{0}}$, there exists $m_{0}>n_{0}$, such that Then from (9) with $x=x_{n}$, and from (10), we have

$$
c \ll p\left(x_{n}, z\right)+p\left(x_{n}, T x_{n}\right)=p\left(x_{n}, z\right)+p\left(x_{n}, x_{n+1}\right) \leq \frac{c}{4}+\frac{c}{4}=\frac{c}{2},
$$

a contradiction, as $c \in \operatorname{int}(P)$ Therefore, our assumption $z \neq T z$ was wrong and so $z=$ $T z$.

If $v=T v$ then we have,

$$
\begin{gathered}
p(v, v)=p\left(T v, T^{2} v\right) \leq k p(v, T v)=k^{n} p(v, v), \\
k^{n} p(v, v)-p(v, v) \in P .
\end{gathered}
$$

and by $\left(\mathbf{p}_{3}\right)$ we have $p(v, v)=0$.

If $v=T v$ then we have, by using (4),

$$
p(v, v)=p\left(T^{n} v, T^{n+1} v\right)=p\left(v_{n}, v_{n+1}\right) \leq k^{n} p(v, T v)=k^{n} p(v, v) .
$$

Hence

$$
k^{n} p(v, v)-p(v, v) \in P .
$$

Since $P$ is closed, by Lemma 2, we get

$$
\lim _{n \rightarrow \infty}\left[k^{n} p(v, v)-p(v, v)\right]=-p(v, v),
$$

and $P$ is closed, we get $-p(v, v) \in P$. Since also $p(v, v) \in P$, then $p(v, v)=0$.

To complete the proof, we have to prove (ii). Suppose that $T$ is continuous. Then from $\left(\mathbf{c}_{1}\right)$, as $\left\{x_{n}\right\}$ converge to $z$, we have

$$
T(z)=\lim _{i \rightarrow \infty} T\left(x_{n}\right)=\lim _{i \rightarrow \infty} x_{n+1}=z .
$$

Thus we proved that $T(z)=z$ and so the proof is complete.

Now we shall present an example where our Theorem 9 can be applied, but the main Theorem 2.2 of Wang and Guo [14] cannot.

Example 10. Let $X=[0,+\infty)$ and $d: X \times X \rightarrow\left(E, \tau^{*}\right)$ be defined by $d(x, y)(t)=\mid x-$ $y \mid . e^{t}$, as in Example 7 above. Then $(X, d)$ is a tvs-cone metric space over the nonnormed linear tvs $\left(E, \tau^{*}\right)$. Define a mapping $T: X \rightarrow X$ by $T x=x / 2$. Then $T$ satisfies the following condition: 


$$
p\left(T x, T^{2} x\right) \leq \frac{1}{2} p(x, T x), \quad \text { for all } \quad x \in X,
$$

and if $y \neq T y$, there exists $c \in \operatorname{int}(P), c \neq \theta$, such that

$$
c \ll p(x, y)+p(x, T x), \text { for all } x \in X .
$$

Thus all hypotheses of our Theorem 9 are satisfied and $z=0$ is a fixed point of $T$. Note that the mapping $T: X \rightarrow X$ satisfies the condition (2.1) in the main Theorem 2.2 of Wang and Guo [14] with $g(x)=x$ and $a_{1}=1 / 2, a_{2}=a_{3}=a_{4}=0$, but Theorem 2.2 cannot be applied since a cone $P$ is not normed.

The following corollary implies the recent result Theorem 3.5. of [4].

Corollary 11. Let $(X, d)$ be a complete tvs-cone metric space with $w$-cone distance $p$ on $X$ and $0 \leq r<1 / 2$. Suppose $T: X \rightarrow X$ and

$$
p\left(T x, T^{2} x\right) \leq r p\left(x, T^{2} x\right), \quad \text { for all } x \in X .
$$

Assume that either (i) or (ii) of Theorem 9 holds. Then, there exists $z \in X$, such that $z$ $=T z$. Moreover, if $v=T v$, then $p(v, v)=\theta$.

Proof. Let $x \in X$. From (12) we have $p\left(T x, T^{2} x\right) \leq r p\left(x, T^{2} x\right) \leq r[p(x, T x)+p(T x$, $\left.T^{2} x\right)$ ]. Hence we get

$$
p\left(T x, T^{2} x\right) \leq k p(x, T x),
$$

where $0 \leq k=r /(1-r)<1$. Now, Corollary 11 follows from Theorem 9 .

If $f: X \rightarrow X$ and $F(f)$ is a set of all fixed points of $f$, then in a general case $F(f) \neq F\left(f^{n}\right)$. Abbas and Rhoades [11] studied cases when $F(f)=F\left(f^{n}\right)$ for each $n \in \mathbb{N}$, that is, when $f$ has a property $P$. The following theorem extends and improves Theorem 2 of [11].

Theorem 12. Let $(X, d)$ be a complete tvs-cone metric space with $w$-cone distance $p$ on $X$. Suppose $T: X \rightarrow X$ satisfies the following condition:

$$
p\left(T x, T^{2} x\right) \leq k p(x, T x), \quad \text { for all } x \in X,
$$

where $0 \leq k<1$, or $T$ satisfies strict the inequality (13) with $k=1$, for all $x \in X$ with $x$ $\neq T x$. If $F(T) \neq \varnothing$, then $T$ has property $P$.

Proof. Let $u \in F\left(T^{n}\right)$ for some $n>1$. Suppose that $T$ satisfies (13). Then

$$
p(u, T u)=p\left(T^{n} u, T T^{n} u\right) \leq k p\left(T^{n-1} u, T T^{n-1} u\right) \leq \cdots \leq k^{n} p(u, T u) .
$$

Similarly as from (11) we get $p(v, v)=\theta$, from (14) we obtain $p(u, T u)=\theta$. Then from (14), $p\left(T^{i} u, T^{i+1} u\right) \leq k^{i} p(u, T u)=\theta$ for all $i \in \mathbb{N}$. Now, from $\left(\mathbf{w}_{1}\right)$ of Definition 4 and $T^{n} u=u$ we get

$$
p(u, u) \leq p(u, T u)+p\left(T u, T^{2} u\right)+\cdots+p\left(T^{n-1} u, T^{n} u\right)=\theta .
$$

Thus $p(u, u)=\theta$. Hence, and by (i) of Lemma 8 with $x=u, y=T u$ and $z=u$, we have $T u=u$. Now suppose that $T$ satisfies strict the inequality (13) with $k=1$ and let $u \in F\left(T^{n}\right)$. If we suppose that $T u \neq u$, then we have $p\left(T u, T^{2} u\right)<p(u, T u)$. If we suppose that $T u=T^{2} u$, then $T^{i} u=T T^{i} u$ for all $i \in N$. Thus we have

$$
p\left(T u, T^{2} u\right)=p\left(T^{2} u, T T^{2} u\right)=\cdots=p\left(T^{n} u, T^{n+1} u\right)=p(u, T u),
$$

a contradiction with $p\left(T u, T^{2} u\right)<p(u, T u)$. Therefore, $T u \neq T^{2} u$. Then we have $p$ $\left(T^{2} u, T^{3} u\right)<p\left(T u, T^{2} u\right)<p(u, T u)$. Continuing this process we obtain 


$$
p(u, T u)=p\left(T^{n} u, T T^{n} u\right)<p\left(T^{n-1} u, T T^{n-1} u\right)<\cdots<p(u, T u),
$$

a contradiction. Therefore, our supposition $T u \neq u$ was wrong. Thus we proved that $F\left(T^{n}\right)=F(T)$.

The following theorem extends Theorem 2.1 of [16] and implies Theorem 3.7 of [17].

Theorem 13. Let $(X, d)$ be a complete tvs-cone metric space with $w$-cone distance $p$ on $X$ and $0 \leq k<1$. Suppose $T: X \rightarrow X$ and there exists an $x \in X$ such that

$$
p\left(T y, T^{2} y\right) \leq k p(y, T y), \quad \text { for all } y \in 0(x, \infty) .
$$

Then,

(i) $\lim T^{n} x=z$ exists and

$$
p\left(T^{n} x, z\right) \leq \frac{k^{n}}{1-k} \cdot p(x, T x) \text { for } n \geq 1 .
$$

(ii) $p(z, T z)=\theta$ if and only if $G(x)=p(x, T x)$ is T-orbitally lower semicontinuous at $z$.

Proof. Observe that (i) follows from the proof of Theorem 9. Now we prove (ii). It is clear that $p(z, T z)=\theta$ implies $G(z)=p(z, T z)=\theta$ and hence $G(z) \leq G\left(x_{n}\right)+\varepsilon$ for any $\varepsilon$ in $E$ with $\theta \ll \varepsilon$ and all $x_{n} \in 0(x, \infty)$. Suppose now that $G$ is $T$-orbitally lower semicontinuous at $z$. Then from $\left(\mathbf{c}_{3}\right)$, as $x_{n}=T^{n} x \rightarrow z$, for any $\varepsilon$ in $E$ with $\theta \ll \varepsilon$ there is $n_{0}$ in $\mathbb{N}$ such that

$$
\theta \leq p(z, T z)=G(z) \leq G\left(x_{n}\right)+\varepsilon=p\left(x_{n}, x_{n+1}\right)+\varepsilon \leq k^{n} p(x, T x)+\frac{1}{i} \varepsilon,
$$

for all $n \geq n_{0}$ and $i \geq 1$. Hence $k^{n} p(x, T x)+\frac{1}{i} \varepsilon-p(z, T z) \in P$. Letting $n \rightarrow \infty$, By Lemma 2, we get $\theta \leq p(z, T z) \leq \frac{1}{i} \varepsilon$. Hence, we get $p(z, T z)=\theta$.

The following theorem extends and unifies Theorem 2 of [6] and results of $([1,18])$.

Theorem 14. Let $(X, d)$ be a complete tvs-cone metric space with $w$-cone distance $p$ on $X$ and $0 \leq k<1$. Suppose that $T: X \rightarrow X$ is a p-contractive mapping i.e.,

$$
p(T x, T y) \leq k p(x, y), \quad \text { for all } x, y \in X .
$$

Then, $T$ has a unique fixed point $z \in X$, and $p(z, z)=\theta$.

Proof. Let $x \in X$ and define $x_{n+1}=T^{n} x$ for any $n \in N$. Then, from the proof of Theorem 9, $\lim T^{n} x=z \in X$ and (3) holds for all $n \geq 1$. From (3) we have

$$
p\left(T^{n} x, z\right) \leq \frac{k^{n}}{1-k} \cdot p(x, T x) \text { for } n \geq 1,
$$

and from (15) and (3) we get

$$
p\left(T^{n} x, T z\right) \leq k p\left(T^{n-1} x, z\right) \leq k \frac{k^{n-1}}{1-k} \cdot p(x, T x)=\frac{k^{n}}{1-k} \cdot p(x, T x) \text { for } \quad n \geq 1 .
$$

Thus from Lemma 2 and (i) of Lemma 8, with $\alpha_{n}=\beta_{n}=\left[k^{n} /(1-k)\right] \cdot p(x, T x)$ we obtain $T z=z$. Then $p(z, z)=p\left(T z, T^{2} z\right) \leq k p(z, T z)=k p(z, z)$. Hence, $p(z, z)=\theta$. Suppose that $u \in X$ is also a fixed point of $T$. Then $p(z, u)=p(T z, T u) \leq k p(z, u)$ and hence $p(z, u)=\theta$. From $p(z, u)=\theta, p(z, z)=\theta$ and by (i) of Lemma 8 , we have $u=z$. 
In 1998, Ume [8] proved the $w$-distance version of Cirić's [19] results for quasi-contraction on metric space. Recently, cone metric version of Ćirić's results has been proved [12]. Now, a natural question arises:

Question. Let $(X, d)$ be a complete tvs-cone metric space with $w$-cone distance $p$ on $X$. Suppose $f: X \mapsto X$ such that for some constant $\lambda \in(0,1)$ and for every $x, y \in X$, there exists $v \in\{p(x, y), p(x, f x), p(y, f y), p(x, f y), p(y, f x)\}$, such that $p(f x, f y) \leq \lambda \cdot v$. Does there exist a unique fixed point $z \in X$ of $f$, and $p(z, z)=\theta$ ?

\section{Acknowledgements}

Authors are supported by Grant No. 174025 of the Ministry of Science, Technology and Development, Republic of Serbia. HL would like to thank Professors Ljubomir Ćirić and Vladimir Rakočević for his valuable help throughout the preparation of this article.

\section{Author details}

${ }^{1}$ Faculty of Mechanical Engineering, Kraljice Marije 16, Belgrade, Serbia ${ }^{2}$ Department of Mathematics, Payame Noor University, 19395-4697 Tehran, Islamic Republic of Iran ${ }^{3}$ Faculty of Sciences and Mathematics, University in Niš, Visegradska 33, Niš, Serbia

\section{Authors' contributions}

All authors contributed equally and significantly in writing this article. All authors read and approved the final manuscript.

\section{Competing interests}

The authors declare that they have no competing interests.

Received: 11 April 2011 Accepted: 4 January 2012 Published: 4 January 2012

\section{References}

1. Huang, LG, Zhang, X: Cone metric spaces and fixed point theorems of contractive mappings. J Math Anal Appl. 332(2):1468-1476 (2007). doi:10.1016/j.jmaa.2005.03.087

2. Du, WS: A note on cone metric fixed point theory and its equivalence. Nonlinear Anal TMA. 72, 2259-2261 (2010). doi:10.1016/j.na.2009.10.026

3. Kada, O, Suzuki, T, Takahashi, W: Nonconvex minimization theorems and fixed point theorems in complete metric spaces. Math Japon. 44, 381-591 (1996)

4. Du, WS: Fixed point theorems for generalized hausdorff metrics. Int Math Forum. 3(21):1011-1022 (2008)

5. Suzuki, T: Several fixed point theorems in complete metric spaces. Yokohama Math J. 44, 61-72 (1997)

6. Suzuki, T, Takahashi, W: Fixed point theorems and characterizations of metric completeness. Topol Methods Nonlinear Anal. 8, 371-382 (1996)

7. Takahashi, W: Minimization theorems and fixed point theorems. In: Maruyama T (ed.) Nonlinear Analysis and Mathematical Economics, vol. 829, pp. 175-191. RIMS Kokuroku

8. Ume, JS: Fixed point theorems related to Ćirić contraction principle. J Math Anal Appl. 225, 630-640 (1998). doi:10.1006/jmaa.1998.6030

9. Beg, I, Azam, A, Arshad, M: Common fixed points for maps on topological vector space valued cone metric spaces. Int J Math Math Sci 8 (2009). doi:10.1155/2009/560264

10. Kadelburg, Z, Radenović, S, Rakočević, V: Topological vector space-valued cone metric spaces and fixed point theorems. Fixed Point Theory Appl (2010). Article ID 170253, 17 (2010). doi:10.1155/2010/170253

11. Abbas, M, Rhoades, BE: Fixed and periodic point results in cone metric spaces. Appl Math Lett. 22, 511-515 (2009). doi:10.1016/j.aml.2008.07.001

12. Gajić, Lj, llić, D, Rakočević, V: On Ćirić maps with a generalized contractive iterate at a point and Fisher's quasicontractions in cone metric spaces. Appl Math Comput. 216, 2240-2247 (2010). doi:10.1016/j.amc.2010.03.010

13. Jungck, G, Radenović, S, Radojević, S, Rakočević, V: Common fixed point theorems for weakly compatible pairs on cone metric spaces. Fixed Point Theory Appl (2009). Article ID 643640, 12 (2009). doi:10.1155/2009/643640

14. Wang, Sh, Guo, B: Distance in cone metric spaces and common fixed point theorems. Appl Math Lett. 24, 1735-1739 (2011). doi:10.1016/j.aml.2011.04.031

15. Vandergraft, JS: Newton's method for convex operators in partially ordered spaces. SIAM J Numer Anal 4, 406-432 (1967). doi:10.1137/0704037. doi:10.1137/0704037

16. Hicks, TL, Rhoades, BE: A Banach type fixed-point theorem. Math Japon. 24, 327-330 (1979)

17. Pathak, HK, Shahzad, N: Fixed point results for generalized quasicontraction mappings in abstract metric spaces. Nonlinear Anal. 71, 6068-6076 (2009). doi:10.1016/j.na.2009.05.052

18. Veazpour, SM, Raja, P: Some extensions of Banach's contraction principle in complete cone metric spaces. Fixed Point Theory Appl (2008). Article ID 768294, 11 (2008). doi:10.1155/2008/768294

19. Ćirić, LjB: A generalization of Banach's contraction principle. Proc Am Math Soc. 45, 267-273 (1974)

doi:10.1186/1687-1812-2012-3

Cite this article as: Ćirić et al:: Fixed point theorems for $w$-cone distance contraction mappings in tvs-cone metric spaces. Fixed Point Theory and Applications 2012 2012:3. 